\title{
Planck's view of the intestellar medium
}

\author{
Planck Collaboration, presented by J. A. Tauber \\ Astrophysics Division, Research and Scientific Support Dpt, European Space Agency, \\ Keplerlaan 1, 2201AZ Noordwijk, The Netherlands \\ email: jtauber@rssd.esa.int
}

\begin{abstract}
Planck is a cosmology experiment, but significant interstellar dust and gas emission exists in the band where the CMB peaks. Therefore, Planck's all-sky surveys provide new views of the ISM and magnetic fields in the Galaxy, as well as the dust and gas in galaxies.
\end{abstract}

Keywords. ISM: general, Galaxy: general, cosmic microwave background, space vehicles

The Planck $\dagger$ satellite (Tauber et al. (2010)), launched on 14 May 2009, has been surveying the sky continuously since 13 August 2009. It carries a scientific payload consisting of an array of detectors arranged in nine broad-band channels sensitive to a range of frequencies between $\sim 25$ and $\sim 1000 \mathrm{GHz}$, which scan the sky simultaneously and continuously with an angular resolution of $5^{\prime}$ to $30^{\prime \prime}$. The Planck satellite, its payload, and its performance at the time of launch are described in Volume 520 of $A \mathscr{E} A$.

The main objective of Planck is to measure the spatial anisotropies of the temperature of the Cosmic Microwave Background (CMB) over the whole sky, with an accuracy set by fundamental astrophysical limits. Its level of performance will enable Planck to extract essentially all the information in the CMB temperature anisotropies. Planck will also measure to high accuracy the polarisation of the CMB anisotropies, which encodes not only a wealth of cosmological information, but also provides a unique probe of the thermal history of the Universe during the time when the first stars and galaxies formed. The scientific objectives of Planck are described in detail in Planck Collaboration (2005).

Planck is by design a cosmology experiment, but very significant Galactic and extragalactic emission exists in the band where the CMB peaks. Thus, its all-sky surveys also produce a wealth of information on the extragalactic sources and on the dust and gas in our own Galaxy. This fact can be clearly appreciated in Fig. 1, which is a composite of data acquired during Plancks first complete all-sky survey. Galactic emission dominates a large part of the sky, both at low frequencies (by a mixture of synchrotron, free-free and other non-thermal radiation) and at high frequencies (mainly by thermal dust emission), and has to be measured accurately and removed to gain access to the CMB.

The Planck survey's frequency range, all-sky coverage, high sensitivity, ability to measure polarization, and high calibration accuracy have enabled numerous ISM studies:

(a) The higher frequency maps allow for the first time to measure accurately the characteristics, amount and distribution of the coldest dust present in the ISM, both in the diffuse ISM (e.g. Planck Early Results XXIV) and in dense molecular clouds (e.g. Planck Early Results XXV), not only in our own Milky Way, but also in nearby objects such as the Magellanic Clouds (Planck Early Results XVII). A spectacular early result by Planck is the detailed mapping of so-called "dark" gas that is not spatially correlated with

$\dagger$ Planck (http://www.esa.int/Planck) is a project of the European Space Agency - ESA - with instruments provided by two scientific Consortia funded by ESA member states (in particular the lead countries: France and Italy) with contributions from NASA (USA), and telescope reflectors provided in a collaboration between ESA and a scientific Consortium led and funded by Denmark. 


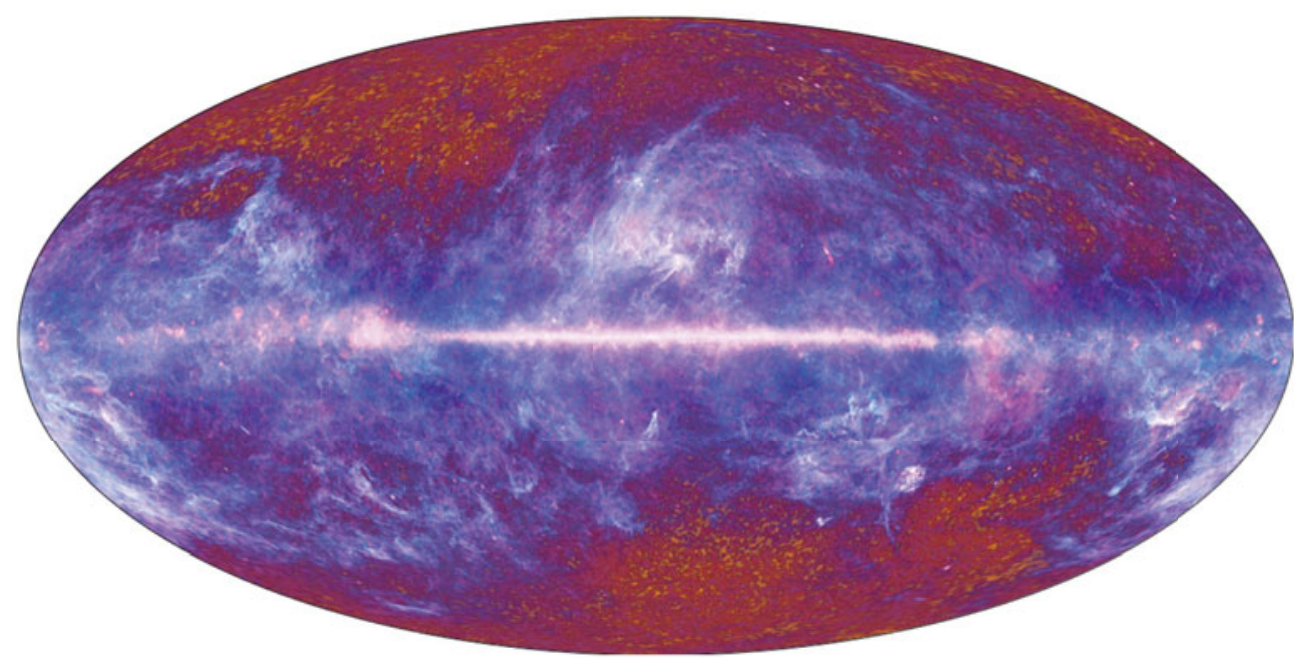

Figure 1. The microwave sky as seen by Planck. This multi-frequency all-sky image of the microwave sky has been composed using data from Planck covering $30 \mathrm{GHz}$ to $857 \mathrm{GHz}$.

known tracers of neutral and molecular gas (Planck Early Results XIX). Plancks ability to detect very cold dust has revealed the widespread presence of dense and compact clumps of gas, certainly the sites of future star formation (Planck Early Results XXIII).

(b) The wide frequency range allows Planck to detect and study components of the ISM with uncommon spectral characteristics, for example the anomalous excess emission possibly arising from small spinning grains (Planck Early Results XX).

(c) The all-sky coverage allows to conduct global studies of the radial distribution of molecular, neutral, and ionised ISM in the Milky Way (Planck Early Results XXI).

(d) The combination of all-sky coverage and cm-to-submm wavelength range allow Planck to survey key parts of the spectral energy distribution of external galaxies (Planck Early Results VII), and has revealed many spectrally flat or rising radio galaxies (Planck Early Results XIII) and a more-than-expected excess of dusty galaxies, due to the detection of previously unaccounted cold dust (Planck Early Results XVI ).

(e) Planck's ability to measure polarization between 30 and $350 \mathrm{GHz}$ promises an exciting magnetic view of the ISM. The Galactic ISM is threaded by magnetic fields whose morphology is largely unknown; this field in turn induces polarization of both synchrotron and dust emission. Planck will uniquely be able to estimate the properties of both the ordered and turbulent components of the Galactic magnetic field.

(f) The extremely accurate calibration of Planck, for both diffuse emissions and compact sources, will provide highly precise photometric standards in this frequency range (Planck Early Results XIV) and Herschel/SPIRE, which is very beneficial to ISM science.

The above examples illustrate the wide range of ISM studies enabled by the Planck. When the Planck data products are released to the public, starting in March 2013, they will join a wide range of surveys from other observatories, which together provide a broad view of the phenomenology of the Interstellar Medium in our own and other galaxies.

\section{References}

Planck Collaboration 2011, A\&A 536, A1

Planck Collaboration 2005, ESA Publication, ESA-SCI(2005)1

Tauber, J. A., Mandolesi, N., Puget, J. L., et al. 2010, A\& A 520, A1 